\title{
ERNST EQUATION, FAY IDENTITIES AND VARIATIONAL FORMULAS ON HYPERELLIPTIC CURVES
}

\author{
C. Klein, D. Korotkin, and V. Shramchenko
}

\begin{abstract}
We present a unified approach to theta-functional solutions of the stationary axisymmetric Einstein equations in vacuum. Using Fay's trisecant identity and variational formulas on hyperelliptic Riemann surfaces, we establish formulas for the metric functions, the Ernst potential and their derivatives.
\end{abstract}

\section{Introduction}

The theory of theta-functional solutions of integrable equations starts in 1974 with the works of Novikov, Dubrovin, Matveev, Its and Krichever (see [1, 2, 3] and references therein) on periodic and quasi-periodic solutions to the Korteweg de Vries (KdV) equation. These finite-gap solutions can be expressed via the Its-Matveev formula [2] in terms of a second derivative of multidimensional theta-functions of a hyperelliptic algebraic curve. The main technical tool exploited in $[1,2]$ was the spectral theory of Sturm-Liouville operators with periodic potential. Later Krichever [3] generalized the Its-Matveev formula to an integrable generalization of the $\mathrm{KdV}$ equation to $2+1$ dimensions - the KadomtzevPetviashvili (KP) equation. Analogous formulas in terms of theta-functions were derived in the framework of the inverse scattering method for other integrable equations as, for example, Sine-Gordon, Non-linear Schrödinger, and LandauLifschitz.

On the other hand $[4,5]$, the finite-gap solutions of integrable systems of KP type may be derived directly from Fay's trisecant identity [6]. This identity holds for any set of four points $(a, b, c, d)$ on a compact Riemann surface $\mathcal{L}$ of genus $g$ and any vector $\mathbf{z} \in \mathbb{C}^{g}$ :

$$
\begin{gathered}
E(c, a) E(d, b) \Theta\left(\mathbf{z}+\int_{b}^{c}\right) \Theta\left(\mathbf{z}+\int_{a}^{d}\right)+E(c, b) E(a, d) \Theta\left(\mathbf{z}+\int_{a}^{c}\right) \Theta\left(\mathbf{z}+\int_{b}^{d}\right) \\
=E(c, d) E(a, b) \Theta(\mathbf{z}) \Theta\left(\mathbf{z}+\int_{b}^{c}+\int_{a}^{d}\right),
\end{gathered}
$$

where $\Theta$ is the theta-function built from the matrix of $b$-periods of the surface $\mathcal{L} ; \int_{b}^{a}$ is a short notation for the difference of Abel maps on $\mathcal{L}$ between points $a$ and $b ; E$ is the prime-form on $\mathcal{L}$ (see sect. 2.1).

Received November 1, 2001. 
Further development of the method of finite-gap integration of integrable systems in [10] allowed to solve in terms of theta-functions the Ernst equation

$$
(\mathcal{E}+\overline{\mathcal{E}})\left(\mathcal{E}_{\zeta \zeta}+\frac{1}{\rho} \mathcal{E}_{\rho}+\mathcal{E}_{\rho \rho}\right)=2\left(\mathcal{E}_{\zeta}^{2}+\mathcal{E}_{\rho}^{2}\right)
$$

for a complex-valued Ernst potential $\mathcal{E}$ depending on two coordinates $(\zeta, \rho)$. The Ernst equation is equivalent to the stationary axially symmetric vacuum Einstein equation; it was embedded in the framework of the inverse scattering method by Belinski-Zakharov [7] and Maison [8] in 1978. In particular, certain multisoliton solutions of the Ernst equation (which form a degenerate subclass of algebrogeometric solutions) give rise to Schwarzschild and Kerr black holes. A class of non-degenerate theta-functional solutions of (1.1) was recently used in $[11,12]$ to describe the gravitational field of rotating dust discs. The theta-functional solutions of (1.1) can be written in the form [14]

$$
\mathcal{E}=\frac{\Theta_{\mathbf{p q}}\left(\int_{\xi}^{\infty^{+}}\right)}{\Theta_{\mathbf{p q}}\left(\int_{\xi}^{\infty^{-}}\right)},
$$

where the theta-function corresponds to the hyperelliptic spectral curve $\mu^{2}=$ $(\lambda-\xi)(\lambda-\bar{\xi}) \prod_{k=1}^{g}\left(\lambda-E_{k}\right)\left(\lambda-F_{k}\right)$, where $\xi=\zeta+i \rho$, and where for each $k$ we require that either $E_{k}=\overline{F_{k}}$ or $E_{k}, F_{k} \in \mathbb{R}$. The constant (with respect to the physical coordinates) vectors $\mathbf{p}$ and $\mathbf{q}$ must satisfy the reality condition $\mathbf{B p}+\mathbf{q} \in \mathbb{R}^{g}$. The notations $\infty^{+}$and $\infty^{-}$are used for the infinite points on different sheets of the curve $\mathcal{L}$, namely, $\mu / \lambda^{g+1} \rightarrow \pm 1$ as $\lambda \rightarrow \infty^{ \pm}$, respectively.

We notice the essential difference between the solution (1.2) of the Ernst equation, and, say, the finite-gap solutions of the KP equation. The spectral curve of the Ernst equation is "dynamical" i.e. it depends on the space-time coordinates $(\xi, \bar{\xi})$, whereas the spectral curve of the KP equation is static, i.e. it is built from the integrals of motion of the system. The dynamical character of the spectral curve of the Ernst equation implies, in particular, the asymptotical flatness of the theta-functional solutions, in contrast to the quasi-periodic nature of previously known theta-functional solutions of equations of KdV and KP type. The solutions (1.1) thus are defined on a family $\mathcal{L}(\xi, \bar{\xi})$ of Riemann surfaces and are in general not periodic or quasi-periodic.

The original derivation of the solutions (1.2) was performed with the use of the zero curvature representation of the Ernst equation, and the solution of an appropriate Riemann-Hilbert problem. The explicit formulas for the coefficients of the space-time metric corresponding to solutions (1.2) required the calculation of the tau-function corresponding to this Riemann-Hilbert problem.

It is the purpose of the present paper to prove the formulas for the Ernst potential (1.2) and the metric functions, by using only the trisecant Fay identity (2.4) (together with its appropriate degenerations) and Rauch's variational formulas [15] which describe the dependence of the holomorphic differentials on 
the moduli of the Riemann surface. Therefore we confirm once more the universality of Fay's identities in the theory of integrable systems, and show their applicability to non-autonomous equations of Ernst type.

In section 2 we collect some useful facts from the theory of Riemann surfaces. In section 3 we prove, using Fay's identity and Rauch's formulas, that the function (1.2) satisfies the Ernst equation. Finally, in section 4 we use the same techniques for formulas for the metric coefficients corresponding to this solution of the Ernst equation. Section 5 contains a summary and an outlook.

\section{Fay's identities and Rauch's variational formulas}

2.1. Fay's identities and their degenerations. Consider a compact Riemann surface $\mathcal{L}$ of genus $g$. On this surface we introduce a canonical basis of cycles $\left(a_{\alpha}, b_{\alpha}\right), \alpha=1, \ldots, g$, the basis of holomorphic differentials normalized

by the condition $\int_{a_{\alpha}} \omega_{\beta}=\delta_{\alpha \beta}$, and the matrix $\mathbf{B}_{\alpha \beta}=\int_{b_{\alpha}} \omega_{\beta}$ of their $b$-periods. The theta-function with characteristics corresponding to the curve $\mathcal{L}$ is given by

$$
\Theta_{\mathbf{p q}}(\mathbf{z} \mid \mathbf{B})=\sum_{\mathbf{m} \in \mathbb{Z}^{g}} \exp \{\pi i\langle\mathbf{B}(\mathbf{p}+\mathbf{m}),(\mathbf{p}+\mathbf{m})\rangle+2 \pi i\langle\mathbf{p}+\mathbf{m}, \mathbf{q}+\mathbf{z}\rangle\}
$$

here $\mathbf{z} \in \mathbb{C}^{g}$ is the argument and $\mathbf{p}, \mathbf{q} \in \mathbb{C}^{g}$ are the vectors of characteristics; $\langle.,$.$\rangle denotes the scalar product. The theta-function with characteristics is, up$ to an exponential factor, equal to the theta-function with zero characteristics (denoted by $\Theta$ ) and shifted argument:

$$
\Theta_{\mathbf{p q}}(\mathbf{z} \mid \mathbf{B})=\Theta(\mathbf{z}+\mathbf{B} \mathbf{p}+\mathbf{q}) \exp \{\pi i\langle\mathbf{B p}, \mathbf{p}\rangle+2 \pi i\langle\mathbf{p}, \mathbf{z}+\mathbf{q}\rangle\}
$$

The theta-function satisfies the heat equation:

$$
4 \pi i \partial_{\mathbf{B}_{\alpha \beta}}\left\{\Theta_{\mathbf{p q}}(\mathbf{z}, \mathbf{B})\right\}=\partial_{z_{\alpha}} \partial_{z_{\beta}} \Theta_{\mathbf{p q}}(\mathbf{z}, \mathbf{B}) .
$$

The main tool we are going to exploit in this paper is Fay's trisecant identity for theta functions and prime forms. The prime form is the $\left(-\frac{1}{2},-\frac{1}{2}\right)$-differential on $\mathcal{L} \times \mathcal{L}$ given by

$$
E(a, b)=\frac{\Theta_{\star}\left(\int_{b}^{a}\right)}{h_{\Delta}(a) h_{\Delta}(b)},
$$

where $h_{\Delta}^{2}(a)=\sum_{\alpha=1}^{g} \frac{\partial \Theta_{\star}}{\partial z_{\alpha}}(0) \omega_{\alpha}\left(\tau_{a}\right)$, and where $\star \equiv\left[\mathbf{p}^{\star} \mathbf{q}^{\star}\right]$ is an odd nonsingular half-integer characteristic (note that the prime form is independent of the choice of the characteristic $\star)$. As before, $\int_{a}^{b}$ denotes the line integral from $a$ to $b$ of the vector $\omega(\tau)=\left(\omega_{1}(\tau), \ldots, \omega_{g}(\tau)\right)^{T}$. 
Fay's trisecant identity holds for any four points $a, b, c, d \in \mathcal{L}$ and any two characteristic vectors $\mathbf{p}, \mathbf{q} \in \mathbb{C}^{g}$ :

$$
\begin{aligned}
& E(c, a) E(d, b) \Theta_{\mathbf{p q}}\left(\mathbf{z}+\int_{b}^{c}\right) \Theta_{\mathbf{p q}}\left(\mathbf{z}+\int_{a}^{d}\right) \\
& +E(c, b) E(a, d) \Theta_{\mathbf{p q}}\left(\mathbf{z}+\int_{a}^{c}\right) \Theta_{\mathbf{p q}}\left(\mathbf{z}+\int_{b}^{d}\right) \\
& =E(c, d) E(a, b) \Theta_{\mathbf{p q}}(\mathbf{z}) \Theta_{\mathbf{p q}}\left(\mathbf{z}+\int_{b}^{c}+\int_{a}^{d}\right) .
\end{aligned}
$$

where all integration contours are chosen not to intersect the canonical basic cycles; this requirement completely fixes all terms of the identity (2.4).

In the sequel we will use the degenerate versions of Fay's identity. Let us denote by $D_{a}$ the operator for the directional derivative along the basis of holomorphic differentials, acting on theta-functions:

$$
D_{a} \Theta_{\mathbf{p q}}(\mathbf{z})=\left\langle\nabla \Theta_{\mathbf{p q}}(\mathbf{z}), \frac{\omega(a)}{d \tau_{a}}\right\rangle \equiv \sum_{\alpha} \partial_{z_{\alpha}}\left\{\Theta_{\mathbf{p q}}(\mathbf{z})\right\} \frac{\omega_{\alpha}(a)}{d \tau_{a}} .
$$

Since the theta-function (2.1) depends only on the sum of vectors $\mathbf{z}$ and $\mathbf{q}$, the action of the operator $D_{a}$ on a theta-function with characteristics can be written alternatively as follows:

$$
D_{a} \Theta_{\mathbf{p q}}(\mathbf{z})=\sum_{\alpha} \partial_{q_{\alpha}}\left\{\Theta_{\mathbf{p q}}(\mathbf{z})\right\} \frac{\omega_{\alpha}(a)}{d \tau_{a}}
$$

This form of $D_{a}$ can be easily extended to any object depending on a vector $\mathbf{q}$.

Differentiating (2.4) with respect to the argument $d$ and taking the limit $d \rightarrow b$ one obtains

Corollary 1. The following degenerated version of Fay's identity holds:

$$
D_{b} \ln \frac{\Theta_{\mathbf{p q}}\left(\mathbf{z}+\int_{a}^{c}\right)}{\Theta_{\mathbf{p q}}(\mathbf{z})}=c_{1}(a, b, c)+c_{2}(a, b, c) \frac{\Theta_{\mathbf{p q}}\left(\mathbf{z}+\int_{a}^{b}\right) \Theta_{\mathbf{p q}}\left(\mathbf{z}+\int_{b}^{c}\right)}{\Theta_{\mathbf{p q}}(\mathbf{z}) \Theta_{\mathbf{p q}}\left(\mathbf{z}+\int_{a}^{c}\right)},
$$

where the functions of three variables $c_{1}$ and $c_{2}$ are given by:

$$
c_{1}(a, b, c)=\frac{\omega_{a, c}(b)}{d \tau_{b}}
$$

where $\omega_{a, c}(b)$ is the differential of the third kind with poles in a, $c$, and

$$
c_{2}(a, b, c)=\frac{E(a, c)}{E(a, b) E(b, c) d \tau_{b}} .
$$

The derivative of (2.6) with respect to argument $c$ gives in the limit $c \rightarrow a$

Corollary 2. The following twice degenerated version of Fay's identity holds:

$$
D_{a} D_{b} \ln \Theta_{\mathbf{p q}}(\mathbf{z})=d_{1}(a, b)+d_{2}(a, b) \frac{\Theta_{\mathbf{p q}}\left(\mathbf{z}+\int_{b}^{a}\right) \Theta_{\mathbf{p q}}\left(\mathbf{z}+\int_{a}^{b}\right)}{\Theta_{\mathbf{p q}}^{2}(\mathbf{z})},
$$


where the functions of two variables $d_{1}$ and $d_{2}$ are given by:

$$
\begin{gathered}
d_{1}(a, b)=-\frac{W(a, b)}{d \tau_{a} d \tau_{b}}, \\
d_{2}(a, b)=\frac{1}{E^{2}(a, b) d \tau_{a} d \tau_{b}} ;
\end{gathered}
$$

$W(a, b)=d_{a} d_{b} \ln E(a, b)$ is the Bergmann kernel.

2.2. Root functions and Rauch's variational formulas on hyperelliptic curves. Let us now choose $\mathcal{L}$ to be the hyperelliptic algebraic curve with $2 g+2$ branch points defined by the equation

$$
\mu^{2}=\prod_{m=1}^{2 g+2}\left(\lambda-\lambda_{m}\right) .
$$

The following identity for "root functions" holds [6] for any point $a \in \mathcal{L}$ :

$$
\frac{E\left(a, \lambda_{m}\right) \sqrt{d \tau_{\lambda_{m}}}}{E\left(a, \lambda_{n}\right) \sqrt{d \tau_{\lambda_{n}}}}=C \sqrt{\frac{\lambda(a)-\lambda_{m}}{\lambda(a)-\lambda_{n}}},
$$

where $\lambda(a)$ denotes the projection of point $a$ onto the Riemann sphere; $C$ is a constant with respect to $\lambda(a)$.

Rauch's variational formulas [15] describe the dependence of the basic normalized holomorphic differentials $\omega_{\alpha}$ and the matrix of $b$-periods $\mathbf{B}_{\alpha \beta}$ on the moduli of the Riemann surface. The moduli space of hyperelliptic curves can be parameterized by the positions of the branch points, and Rauch's formulas read:

$$
\begin{aligned}
\frac{d \omega_{\alpha}}{d \lambda_{m}}(a) & =\frac{1}{2} \frac{W\left(a, \lambda_{m}\right)}{d \tau_{\lambda_{m}}} \frac{\omega_{\alpha}\left(\lambda_{m}\right)}{d \tau_{\lambda_{m}}}, \\
\frac{d \mathbf{B}_{\alpha \beta}}{d \lambda_{m}} & =\pi i \frac{\omega_{\alpha}\left(\lambda_{m}\right)}{d \tau_{\lambda_{m}}} \frac{\omega_{\beta}\left(\lambda_{m}\right)}{d \tau_{\lambda_{m}}},
\end{aligned}
$$

The formulas (2.14), (2.15), together with the heat equation for thetafunctions (2.3), imply the following dependence of hyperelliptic theta-functions on the branch points:

Lemma 1. The derivative of the hyperelliptic theta-function $\Theta_{\mathbf{p q}}(\mathbf{z})$ with a $\left\{\lambda_{m}\right\}$-dependent argument $\mathbf{z}$ with respect to a branch point $\lambda_{m}$ is given by

$$
\partial_{\lambda_{m}} \Theta_{\mathbf{p q}}(\mathbf{z})=\frac{1}{4} D_{\lambda_{m}} D_{\lambda_{m}} \Theta_{\mathbf{p q}}(\mathbf{z})+\sum_{\alpha} \partial_{z_{\alpha}}\left\{\Theta_{\mathbf{p q}}(\mathbf{z})\right\} \frac{d \mathbf{z}_{\alpha}}{d \lambda_{m}}
$$




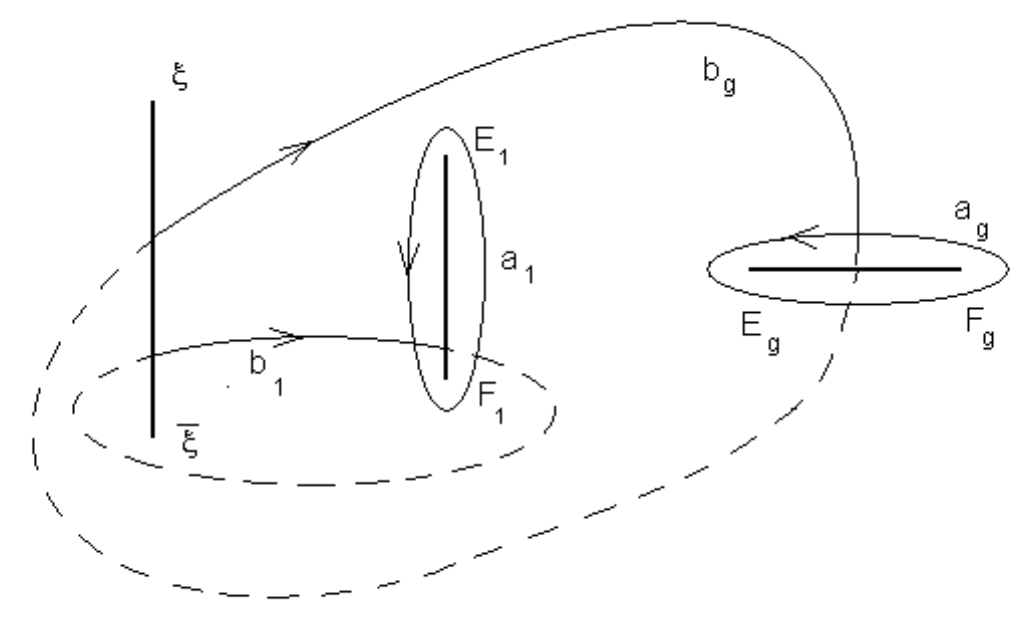

Figure 1.

\section{Ernst equation and Fay identities}

Consider a real hyperelliptic Riemann surface $\mathcal{L}$ of genus $g$ given by

$$
\mu^{2}=(\lambda-\xi)(\lambda-\bar{\xi}) \prod_{m=1}^{g}\left(\lambda-E_{m}\right)\left(\lambda-F_{m}\right),
$$

where $\xi=\zeta-i \rho ; \zeta, \rho \in \mathbb{R}$; for each $m$ we require that either $E_{m}=\overline{F_{m}}$ or $E_{m}, F_{m} \in \mathbb{R}$. Let us introduce the canonical basis of cycles on $\mathcal{L}$ according to Figure 1 above, the $a$-cycles are chosen to encircle the branch cuts $\left[E_{m}, F_{m}\right]$; $b$-cycles all start at the branch cut $[\xi, \bar{\xi}]$. In the sequel we shall denote the point which belongs to the upper sheet of $\mathcal{L}$ and has the projection $\lambda$ on $\mathbb{C P} 1$ by $\lambda^{+}$; the point which has the same projection on $\mathbb{C P} 1$ but belongs to the lower sheet will be denoted by $\lambda^{-}$.

It is convenient to rewrite the Ernst equation (1.1) in terms of the complex coordinates $(\xi, \bar{\xi})$ as follows:

$$
(\mathcal{E}+\overline{\mathcal{E}})\left(\mathcal{E}_{\xi \bar{\xi}}-\frac{1}{2(\bar{\xi}-\xi)}\left(\mathcal{E}_{\bar{\xi}}-\mathcal{E}_{\xi}\right)\right)=2 \mathcal{E}_{\xi} \mathcal{E}_{\bar{\xi}}
$$

This section will be devoted to the proof of the following theorem using Fay's identities and Rauch's formulas:

Theorem 1. Let the branch points $E_{m}, F_{m}$ of the curve $\mathcal{L}$ (3.1) be $(\xi, \bar{\xi})$ independent. Then the function

$$
\mathcal{E}=\frac{\Theta_{\mathbf{p q}}\left(\int_{\xi}^{\infty^{+}}\right)}{\Theta_{\mathbf{p q}}\left(\int_{\xi}^{\infty^{-}}\right)}
$$


where the theta-function corresponds to the matrix of b-periods of the curve $\mathcal{L}$, and where an arbitrary $(\xi, \bar{\xi})$-independent non-singular characteristic $[\mathbf{p}, \mathbf{q}]$ obeys the reality conditions

$$
\mathbf{B p}+\mathbf{q} \in \mathbb{R}^{g},
$$

satisfies the Ernst equation (3.2) in the region of the $\xi$-plane, where the vector $\mathbf{B p}+\mathbf{q}$ does not belong to the theta-divisor on the Jacobi manifold of $\mathcal{L}$ (i.e. $\left.\Theta_{\mathbf{p q}}(0) \neq 0\right)$, and, in addition,

$$
\Theta_{\mathbf{p q}}\left(\int_{\xi}^{\infty^{-}}\right) \neq 0
$$

In accordance with the previous notation, $\int_{\xi}^{a}$ denotes the line integral of the vector $\omega=\left(\omega_{1}, \ldots, \omega_{g}\right)^{T}$ from $\xi$ to a. The integration paths in the numerator and denominator are supposed to have the same projection onto $\mathbb{C P} 1$; therefore, $\int_{\xi}^{\infty^{+}}=-\int_{\xi}^{\infty^{-}}$.

The proof will consist of a series of auxiliary statements: we shall compute the derivatives of the Ernst potential with respect to $(\xi, \bar{\xi})$ and the action of the cylindrical Laplace operator

$$
\Delta \equiv \partial_{\rho \rho}^{2}+\frac{1}{\rho} \partial_{\rho}+\partial_{\zeta \zeta}^{2} \equiv 4\left(\partial_{\xi \bar{\xi}}^{2}-\frac{1}{2(\bar{\xi}-\xi)}\left(\partial_{\bar{\xi}}-\partial_{\xi}\right)\right)
$$

on the Ernst potential. We note that the real part of the Ernst potential can be written in a compact form:

Proposition 1. The real part of the Ernst potential is given by the following expression:

$$
\mathcal{E}+\overline{\mathcal{E}}=2 Q \frac{\Theta_{\mathbf{p q}}(0) \Theta_{\mathbf{p q}}\left(\int_{\bar{\xi}}^{\xi}\right)}{\Theta_{\mathbf{p q}}\left(\int_{\xi}^{\infty^{-}}\right) \Theta_{\mathbf{p q}}\left(\int_{\bar{\xi}}^{\infty^{-}}\right)}
$$

where the function

$$
Q(\xi, \bar{\xi})=\frac{1}{2} \frac{E(\xi, \bar{\xi}) E\left(\infty^{-}, \infty^{+}\right)}{E\left(\xi, \infty^{-}\right) E\left(\bar{\xi}, \infty^{+}\right)}
$$

does not depend on $\mathbf{p}, \mathbf{q}$. Taking into account that $\mathcal{E} \equiv 1$ if $\mathbf{p}=\mathbf{q}=0$, we get an alternative form of the function $Q$ in terms of theta-functions with zero characteristics $\left(\Theta \equiv \Theta_{\mathbf{0 0}}\right)$ :

$$
Q=\frac{\Theta\left(\int_{\xi}^{\infty^{-}}\right) \Theta\left(\int_{\bar{\xi}}^{\infty^{-}}\right)}{\Theta(0) \Theta\left(\int_{\xi}^{\bar{\xi}}\right)} .
$$

Proof. The proof is an immediate corollary of Fay's identity (2.4) applied to the points $\left(\infty^{+}, \infty^{-}, \xi, \bar{\xi}\right)$ if we note the following 
Lemma 2. The following relation holds:

$$
\frac{E\left(\infty^{+}, \bar{\xi}\right) E\left(\infty^{-}, \xi\right)}{E\left(\infty^{-}, \bar{\xi}\right) E\left(\infty^{+}, \xi\right)}=-1 .
$$

Proof. To prove (3.8) we use formula ([6], p.21) which is valid for arbitrary four points $a, b, c, d$ on $\mathcal{L}$ :

$$
\ln \frac{E(b, d) E(a, c)}{E(a, d) E(b, c)}=\int_{c}^{d} \omega_{b, a}
$$

where $\omega_{b, a}$ is normalized (all $a$-periods vanish) differential of the third kind on $\mathcal{L}$ with poles at $a$ and $b$ and residues -1 and +1 , respectively. Assuming $a=\bar{\xi}, b=\xi, c=\infty^{-}, d=\infty^{+}$, we get the integral $\int_{\infty^{-}}^{\infty^{+}} \omega_{\xi, \bar{\xi}}$ along the path encircling the branch point $\xi$. On the hyperelliptic curve (3.1) with our choice of canonical cycles (Fig.1) the abelian integral $\int \omega_{\xi, \bar{\xi}}$ can be computed explicitly to give $\frac{1}{2} \ln \frac{\lambda-\xi}{\lambda-\bar{\xi}}+C$, where $C$ is an arbitrary constant (indeed, this expression has the required structure of singularities at $\xi$ and $\bar{\xi}$, and does not suffer any modification with respect to tracing along $a$-cycles shown in Fig.1; we remind that the local parameters around $\xi$ and $\bar{\xi}$ are $\sqrt{\lambda-\xi}$ and $\sqrt{\lambda-\bar{\xi}}$, respectively). Therefore,

$$
\int_{\infty^{-}}^{\infty^{+}} \omega_{\xi, \bar{\xi}}=\left.\frac{1}{2} \ln \frac{\lambda-\xi}{\lambda-\bar{\xi}}\right|_{\infty^{+}} ^{\infty^{-}}=\frac{1}{2} 2 \pi i=\pi i,
$$

which gives (3.8).

3.1. First derivatives of the Ernst potential. We will first give convenient relations for the first derivatives of the Ernst potential which were obtained in [13] with the use of the zero-curvature representation of the Ernst equation.

Proposition 2. The first derivatives of the Ernst potential (3.3) are given by the following expressions:

$$
\begin{gathered}
\mathcal{E}_{\xi}=\frac{c_{2}\left(\infty^{-}, \xi, \infty^{+}\right)}{2} \frac{\Theta_{\mathbf{p q}}(0)}{\Theta_{\mathbf{p q}}^{2}\left(\int_{\xi}^{\infty^{-}}\right)} D_{\xi} \Theta_{\mathbf{p q}}(0) . \\
\mathcal{E}_{\bar{\xi}}=\frac{c_{2}\left(\infty^{-}, \bar{\xi}, \infty^{+}\right)}{2} \frac{\Theta_{\mathbf{p q}}\left(\int_{\bar{\xi}}^{\xi}\right)}{\Theta_{\mathbf{p q}}^{2}\left(\int_{\xi}^{\infty^{-}}\right)} D_{\bar{\xi}} \Theta_{\mathbf{p q}}\left(\int_{\xi}^{\bar{\xi}}\right),
\end{gathered}
$$

where $c_{2}$ is the constant (2.8) from the degenerated Fay identity (2.6).

Proof. Let us first note the following corollary of Rauch's variational formulas:

$$
\frac{d}{d \xi} \int_{\xi}^{\infty^{+}} \omega_{\alpha}(\tau) \equiv-\frac{d}{d \xi} \int_{\xi}^{\infty^{-}} \omega_{\alpha}(\tau)=-\frac{1}{4} c_{1}\left(\infty^{-}, \xi, \infty^{+}\right) \frac{\omega_{\alpha}(\xi)}{d \tau_{\xi}} .
$$

where $c_{1}$ is as defined in (2.7). To prove (3.12) we notice that, according to (2.14), the derivative of a holomorphic differential with respect to a branch point 
is proportional to the normalized differential of the second kind (the Bergmann kernel); consequently the integration of this differential gives a differential of the third kind, according to (2.7), (3.12).

The idea of the proof is to differentiate the Ernst potential with respect to $\xi$ and to use (2.16) and (3.12) to relate these derivatives to directional derivatives of the theta functions. We get

$$
(\ln \mathcal{E})_{\xi}=\frac{1}{4}\left\{D_{\xi} D_{\xi} \ln \mathcal{E}+\left(D_{\xi} \ln \Theta_{\mathbf{p q}}\left(\int_{\xi}^{\infty^{+}}\right)\right)^{2}\right.
$$

$$
\left.-\left(D_{\xi} \ln \Theta_{\mathbf{p q}}\left(\int_{\xi}^{\infty^{-}}\right)\right)^{2}-c_{1}\left(\infty^{-}, \xi, \infty^{+}\right) D_{\xi} \ln \left(\Theta_{\mathbf{p q}}\left(\int_{\xi}^{\infty^{+}}\right) \Theta_{\mathbf{p q}}\left(\int_{\xi}^{\infty^{-}}\right)\right)\right\}
$$

The resulting expression can be simplified with the help of Fay's identities. It follows from Fay's identity (2.6) with $\mathbf{z}=\int_{\xi}^{\infty^{-}}, a=\infty^{-}, b=\xi, c=\infty^{+}$that $^{1}$

$$
D_{\xi} \ln \mathcal{E}=c_{1}\left(\infty^{-}, \xi, \infty^{+}\right)+c_{2}\left(\infty^{-}, \xi, \infty^{+}\right) \frac{\Theta_{\mathbf{p q}}^{2}(0)}{\Theta_{\mathbf{p q}}\left(\int_{\xi}^{\infty^{-}}\right) \Theta_{\mathbf{p q}}\left(\int_{\xi}^{\infty^{+}}\right)} ;
$$

applying the operator $D_{\xi}$ once more to both sides of this identity, we get

$$
D_{\xi} D_{\xi} \ln \mathcal{E}=c_{2}\left(\infty^{-}, \xi, \infty^{+}\right) D_{\xi}\left\{\frac{\Theta_{\mathbf{p q}}^{2}(0)}{\Theta_{\mathbf{p q}}\left(\int_{\xi}^{\infty^{-}}\right) \Theta_{\mathbf{p q}}\left(\int_{\xi}^{\infty^{+}}\right)}\right\} .
$$

Substituting this expression into (3.13), we arrive at the formula

$$
\begin{gathered}
(\ln \mathcal{E})_{\xi}=\frac{1}{4} c_{2}\left(\infty^{-}, \xi, \infty^{+}\right) D_{\xi}\left\{\frac{\Theta_{\mathbf{p q}}^{2}(0)}{\Theta_{\mathbf{p q}}\left(\int_{\xi}^{\infty^{-}}\right) \Theta_{\mathbf{p q}}\left(\int_{\xi}^{\infty^{+}}\right)}\right\} \\
+\frac{1}{4} D_{\xi} \ln \left\{\Theta_{\mathbf{p q}}\left(\int_{\xi}^{\infty^{+}}\right) \Theta_{\mathbf{p q}}\left(\int_{\xi}^{\infty^{-}}\right)\right\}\left\{D_{\xi} \ln \mathcal{E}-c_{1}\left(\infty^{-}, \xi, \infty^{+}\right)\right\} .
\end{gathered}
$$

We use (3.14) again to simplify the last term. The result is

$$
(\ln \mathcal{E})_{\xi}=\frac{c_{2}\left(\infty^{-}, \xi, \infty^{+}\right)}{2} \frac{\Theta_{\mathbf{p q}}(0) D_{\xi} \Theta_{\mathbf{p q}}(0)}{\Theta_{\mathbf{p q}}\left(\int_{\xi}^{\infty^{-}}\right) \Theta_{\mathbf{p q}}\left(\int_{\xi}^{\infty^{+}}\right)},
$$

which is equivalent to (3.10). The expression (3.11) for $\mathcal{E}_{\bar{\xi}}$ can be proved analogously.

\footnotetext{
${ }^{1}$ It is worth noticing at this point that the action of the operator $D_{\xi}$ on the Ernst potential has a priori nothing to do with the partial derivative of the Ernst potential with respect to $\xi$ : according to the definition $(2.5), D_{\xi} \mathcal{E}$ is just a directional derivative of $\mathcal{E}$ with respect to $\mathbf{q}$ in the direction given by the values of the basic holomorphic differentials at the branch point $\xi$ of the Riemann surface $\mathcal{L}$.
} 
3.2. Action of the Laplace operator on the Ernst potential. The same techniques can be used to determine the second derivatives of the Ernst potential which enter the axisymmetric Laplace operator.

Theorem 2. The action of the cylindrical Laplace operator (3.4) on the Ernst potential has the following form:

$$
\Delta \mathcal{E}=-2 c_{2}\left(\infty^{-}, \xi, \infty^{+}\right) c_{2}\left(\xi, \bar{\xi}, \infty^{+}\right) \frac{\Theta_{\mathbf{p q}}\left(\int_{\bar{\xi}}^{\infty^{-}}\right)}{\Theta_{\mathbf{p q}}^{3}\left(\int_{\xi}^{\infty^{-}}\right)} D_{\bar{\xi}} \Theta_{\mathbf{p q}}\left(\int_{\xi}^{\bar{\xi}}\right) D_{\xi} \Theta_{\mathbf{p q}}(0)
$$

where the ratio of the prime-forms $c_{2}$ is defined by (2.8).

To prove (3.16) we need to compute the derivatives with respect to $\bar{\xi}$ of all three multipliers in (3.10) with the help of the degenerated versions (2.6) and (2.9) of Fay's identities. These derivatives are given by the following three propositions.

Proposition 3. The following identity holds:

$$
\begin{aligned}
& 4\left\{\ln \frac{\Theta_{\mathbf{p q}}(0)}{\Theta_{\mathbf{p q}}\left(\int_{\xi}^{\infty^{-}}\right)}\right\}_{\bar{\xi}}=-c_{2}^{2}\left(\xi, \bar{\xi}, \infty^{-}\right)+c_{1}^{2}\left(\xi, \bar{\xi}, \infty^{-}\right) \\
& -2 c_{2}\left(\xi, \bar{\xi}, \infty^{-}\right) D_{\bar{\xi}} \ln \Theta_{\mathbf{p q}}\left(\int_{\xi}^{\bar{\xi}}\right) \frac{\Theta_{\mathbf{p q}}\left(\int_{\xi}^{\bar{\xi}}\right) \Theta_{\mathbf{p q}}\left(\int_{\bar{\xi}}^{\infty^{-}}\right)}{\Theta_{\mathbf{p q}}\left(\int_{\xi}^{\infty^{-}}\right) \Theta_{\mathbf{p q}}(0)} .
\end{aligned}
$$

Proof. Using identity (2.16) as before, we can write down the l.h.s. of (3.17) as

$$
\begin{gathered}
D_{\bar{\xi}} D_{\bar{\xi}} \ln \frac{\Theta_{\mathbf{p q}}(0)}{\Theta_{\mathbf{p q}}\left(\int_{\xi}^{\infty^{-}}\right)}+D_{\bar{\xi}} \ln \left\{\Theta_{\mathbf{p q}}(0) \Theta_{\mathbf{p q}}\left(\int_{\xi}^{\infty^{-}}\right)\right\} D_{\bar{\xi}} \ln \frac{\Theta_{\mathbf{p q}}(0)}{\Theta_{\mathbf{p q}}\left(\int_{\xi}^{\infty^{-}}\right)} \\
-c_{1}\left(\infty^{-}, \xi, \infty^{+}\right) D_{\bar{\xi}} \ln \Theta_{\mathbf{p q}}\left(\int_{\xi}^{\infty^{-}}\right) .
\end{gathered}
$$

Using the once degenerated Fay identity (2.6) twice, we transform this expression to

$$
\begin{aligned}
&-c_{2}\left(\xi, \bar{\xi}, \infty^{-}\right) \frac{D_{\bar{\xi}}\left(\Theta_{\mathbf{p q}}\left(\int_{\xi}^{\bar{\xi}}\right) \Theta_{\mathbf{p q}}\left(\int_{\bar{\xi}}^{\infty^{-}}\right)\right)}{\Theta_{\mathbf{p q}}(0) \Theta_{\mathbf{p q}}\left(\int_{\bar{\xi}}^{\infty^{-}}\right)} \\
&+\left(-c_{1}\left(\infty^{-}, \bar{\xi}, \infty^{+}\right)-c_{1}\left(\xi, \bar{\xi}, \infty^{-}\right)\right) D_{\bar{\xi}} \ln \Theta_{\mathbf{p q}}\left(\int_{\xi}^{\infty^{-}}\right) \\
&-c_{1}\left(\xi, \bar{\xi}, \infty^{-}\right) D_{\bar{\xi}} \ln \Theta_{\mathbf{p q}}(0) .
\end{aligned}
$$

Since it follows directly from the definition (2.7) of the function $c_{1}$ that

$$
c_{1}\left(\infty^{-}, \bar{\xi}, \infty^{+}\right)=-2 c_{1}\left(\xi, \bar{\xi}, \infty^{-}\right)
$$


the last two terms in (3.18) can be combined, which leads to

$$
\begin{gathered}
-c_{2}\left(\xi, \bar{\xi}, \infty^{-}\right) \frac{\Theta_{\mathbf{p q}}\left(\int_{\xi}^{\bar{\xi}}\right) \Theta_{\mathbf{p q}}\left(\int_{\bar{\xi}}^{\infty^{-}}\right)}{\Theta_{\mathbf{p q}}(0) \Theta_{\mathbf{p q}}\left(\int_{\xi}^{\infty^{-}}\right)}\left(2 D_{\bar{\xi}} \ln \Theta_{\mathbf{p q}}\left(\int_{\xi}^{\bar{\xi}}\right)+D_{\bar{\xi}} \ln \frac{\Theta_{\mathbf{p q}}\left(\int_{\bar{\xi}}^{\infty^{-}}\right)}{\Theta_{\mathbf{p q}}\left(\int_{\bar{\xi}}^{\xi}\right)}\right) \\
+c_{1}\left(\xi, \bar{\xi}, \infty^{-}\right) D_{\bar{\xi}} \ln \frac{\Theta_{\mathbf{p q}}\left(\int_{\xi}^{\infty^{-}}\right)}{\Theta_{\mathbf{p q}}(0)} .
\end{gathered}
$$

We can use Fay's identities to further simplify (3.20). The idea is to eliminate all derivatives of theta functions except of those with argument $\int_{\xi}^{\bar{\xi}}$. For the second term in the first line we apply (2.6) with $\mathbf{z}=\int_{\bar{\xi}}^{\xi}, a=\xi, b=\bar{\xi}, c=\infty^{-}$, for the last term we use the same identity with $a=\xi, b=\bar{\xi}, c=\infty^{-}$. Subsequent simplification of the obtained expression leads to (3.17).

The next proposition gives the $\bar{\xi}$-derivative of the second multiplier in (3.10):

Proposition 4. The following relation holds:

$$
2\left(D_{\xi} \ln \Theta_{\mathbf{p q}}(0)\right)_{\bar{\xi}}=d_{2}(\bar{\xi}, \xi) \frac{\Theta_{\mathbf{p q}}\left(\int_{\bar{\xi}}^{\xi}\right)}{\Theta_{\mathbf{p q}}^{2}(0)} D_{\bar{\xi}} \Theta_{\mathbf{p q}}\left(\int_{\xi}^{\bar{\xi}}\right) .
$$

Proof. Using (2.16) and (2.14), we get

$$
\begin{aligned}
\left(D_{\xi} \ln \Theta_{\mathbf{p q}}(0)\right)_{\bar{\xi}}= & \frac{1}{4} D_{\xi}\left(D_{\bar{\xi}} D_{\bar{\xi}} \ln \Theta_{\mathbf{p q}}(0)+\left(D_{\bar{\xi}} \ln \Theta_{\mathbf{p q}}(0)\right)^{2}\right) \\
& +\frac{1}{2} \frac{W(\xi, \bar{\xi})}{d \tau_{\xi} d \tau_{\bar{\xi}}} D_{\bar{\xi}} \ln \Theta_{\mathbf{p q}}(0) .
\end{aligned}
$$

Applying the twice degenerated Fay identity (2.9) and its $D_{\bar{\xi}}$-derivative to the different terms of this expression, and taking into account that $\frac{W(\xi, \bar{\xi})}{d \tau_{\xi} d \tau_{\bar{\xi}}}=-d_{1}(\xi, \bar{\xi})$, we transform this expression to the r.h.s. of (3.21).

The subsequent statement provides the expression for the $\bar{\xi}$-derivative of the third term in (3.10):

Proposition 5. The following relation holds:

$$
\partial_{\bar{\xi}} \ln c_{2}\left(\infty^{-}, \xi, \infty^{+}\right)=-\frac{1}{2}\left(c_{1}^{2}\left(\xi, \bar{\xi}, \infty^{-}\right)-c_{2}^{2}\left(\xi, \bar{\xi}, \infty^{-}\right)\right)-\frac{1}{2(\bar{\xi}-\xi)}
$$

Proof. In the proof we shall need a corollary of formula (2.13):

Lemma 3. The following relation holds:

$$
\frac{ \pm 1}{\sqrt{\xi-\bar{\xi}}}=\frac{c_{2}\left(\infty^{-}, \xi, \infty^{+}\right)}{2 Q}=c_{2}\left(\bar{\xi}, \xi, \infty^{+}\right)
$$


The correct sign in (3.23) depends on the choice of all branches of the square roots in (3.23) and is unessential for our purposes.

Proof. To prove (3.23) it is sufficient to consider the ratio of two root functions (2.13): one with $\lambda_{n}=\xi, \lambda_{m}=\bar{\xi}$, and $a=\infty^{+}$and another with $\lambda_{n}=\xi, \lambda_{m}=\bar{\xi}$ and $a \rightarrow \lambda_{m}$. Then the unknown function $C$ in (2.13) drops out and we end up with (3.23).

Relation (3.23) implies

$$
\left.\left(\ln c_{2}\left(\infty^{-}, \xi, \infty^{+}\right)\right)\right)_{\bar{\xi}}=(\ln Q)_{\bar{\xi}}+\frac{1}{2(\xi-\bar{\xi})} .
$$

Now we shall prove that for the function $Q(\xi, \bar{\xi})$ given by (3.7)

$$
2(\ln Q)_{\bar{\xi}}=c_{2}^{2}\left(\xi, \bar{\xi}, \infty^{-}\right)-c_{1}^{2}\left(\xi, \bar{\xi}, \infty^{-}\right) .
$$

It is convenient to use the representation of $Q$ in terms of theta functions with zero characteristics (3.7):

$$
(\ln Q)_{\bar{\xi}}=\left(\ln \frac{\Theta\left(\int_{\xi}^{\infty^{-}}\right)}{\Theta(0)}\right)_{\bar{\xi}}+\left(\ln \frac{\Theta\left(\int_{\bar{\xi}}^{\infty^{-}}\right)}{\Theta\left(\int_{\bar{\xi}}^{\bar{\xi}}\right)}\right)_{\bar{\xi}} .
$$

Using the result of proposition 3 with $\mathbf{p}=\mathbf{q}=0$, we see that

$$
\left(\ln \frac{\Theta\left(\int_{\xi}^{\infty^{-}}\right)}{\Theta(0)}\right)_{\bar{\xi}}=\frac{1}{4}\left(c_{2}^{2}\left(\xi, \bar{\xi}, \infty^{-}\right)-c_{1}^{2}\left(\xi, \bar{\xi}, \infty^{-}\right)\right),
$$

since $D_{\bar{\xi}} \ln \Theta\left(\int_{\xi}^{\bar{\xi}}\right)$ vanishes being a directional derivative at zero of an even function. In the same way one can prove that

$$
\left(\ln \frac{\Theta\left(\int_{\bar{\xi}}^{\infty^{-}}\right)}{\Theta\left(\int_{\xi}^{\bar{\xi}}\right)}\right)_{\bar{\xi}}=\frac{1}{4}\left(c_{2}^{2}\left(\xi, \bar{\xi}, \infty^{-}\right)-c_{1}^{2}\left(\xi, \bar{\xi}, \infty^{-}\right)\right) .
$$

Propositions 3, 4 and 5 lead to (3.16) if we take into account the next lemma:

Lemma 4. The following identity holds:

$$
\frac{1}{\xi-\bar{\xi}} \frac{c_{2}\left(\infty^{-}, \bar{\xi}, \infty^{+}\right)}{c_{2}\left(\infty^{-}, \xi, \infty^{+}\right)}+d_{2}(\bar{\xi}, \xi)=0
$$

Proof. We rewrite the left hand side in prime forms, using (3.23) for $(\xi-\bar{\xi})$. Then

$$
\begin{aligned}
\frac{1}{\xi-\bar{\xi}} \frac{c_{2}\left(\infty^{-}, \bar{\xi}, \infty^{+}\right)}{c_{2}\left(\infty^{-}, \xi, \infty^{+}\right)} & =\frac{E\left(\infty^{+}, \bar{\xi}\right)}{E^{2}(\bar{\xi}, \xi) E\left(\infty^{+}, \xi\right) d \tau_{\xi}} \frac{E\left(\infty^{-}, \xi\right)}{E\left(\infty^{-}, \bar{\xi}\right) d \tau_{\bar{\xi}}} \\
= & -\frac{1}{E^{2}(\xi, \bar{\xi}) d \tau_{\xi} d \tau_{\bar{\xi}}},
\end{aligned}
$$


here we used that $\int_{\xi}^{\infty^{+}}=-\int_{\xi}^{\infty^{-}}$and $\int_{\bar{\xi}}^{\infty^{+}}=-\int_{\bar{\xi}}^{\infty^{-}}$and took into account that the prime form is proportional to a theta-function with odd characteristic. The minus sign in (3.27) appears due to lemma 2.

3.3. The Ernst equation. To verify that (3.3) is a solution of the Ernst equation, one has to compare the action (3.16) of the Laplace operator on the Ernst potential with the expression

$$
\frac{8 \mathcal{E}_{\xi} \mathcal{E}_{\bar{\xi}}}{\mathcal{E}+\overline{\mathcal{E}}}=\frac{c_{2}\left(\infty^{-}, \xi, \infty^{+}\right) c_{2}\left(\infty^{-}, \bar{\xi}, \infty^{+}\right)}{Q} \frac{\Theta_{\mathbf{p q}}\left(\int_{\bar{\xi}}^{\infty^{-}}\right)}{\Theta_{\mathbf{p q}}^{3}\left(\int_{\xi}^{\infty^{-}}\right)} D_{\xi} \Theta_{\mathbf{p q}}(0) D_{\bar{\xi}} \Theta_{\mathbf{p q}}\left(\int_{\xi}^{\bar{\xi}}\right)
$$

computed from (3.10), (3.11) and (3.5). The coincidence of these terms follows from the definitions of $c_{2}$ and $Q$.

\section{Metric functions for the stationary axisymmetric vacuum}

The metric of the stationary axisymmetric vacuum spacetimes can be written in the Weyl-Lewis-Papapetrou form (see [9])

$$
\mathrm{d} s^{2}=-e^{2 U}(\mathrm{~d} t+A \mathrm{~d} \phi)^{2}+e^{-2 U}\left(e^{2 k}\left(\mathrm{~d} \rho^{2}+\mathrm{d} \zeta^{2}\right)+\rho^{2} \mathrm{~d} \phi^{2}\right)
$$

where $\rho$ and $\zeta$ are Weyl's canonical coordinates and $\partial_{t}$ and $\partial_{\phi}$ are the commuting asymptotically timelike respectively spacelike Killing vectors.

In this case the vacuum field equations are equivalent to the Ernst equation (1.1) for the complex potential $\mathcal{E}$. For a given Ernst potential, the metric (4.1) can be constructed as follows: the metric function $e^{2 U}$ is equal to the real part of the Ernst potential, which can be written in the form (3.5). The functions $A$ and $k$ can be obtained via a line integration from the equations

$$
A_{\xi}=2 \rho \frac{(\mathcal{E}-\overline{\mathcal{E}})_{\xi}}{(\mathcal{E}+\overline{\mathcal{E}})^{2}}
$$

and

$$
k_{\xi}=(\xi-\bar{\xi}) \frac{\mathcal{E}_{\xi} \overline{\mathcal{E}}_{\xi}}{(\mathcal{E}+\overline{\mathcal{E}})^{2}}
$$

Explicit integration of equations (4.2) and (4.3) is rather non-trivial; for the algebro-geometric solutions (1.2) it was carried out explicitly, exploiting the zerocurvature representation, in the papers $[10,14,13]$. In the sequel we show how to achieve these results on the sole base of Fay's identities and Rauch's formulas.

4.1. Metric function $A$. It was shown in [10] with the help of the inverse scattering method that the function $A$, corresponding to the Ernst potential (2.12), is related to a logarithmic derivative of theta functions which was alternatively expressed in [13] via theta functions themselves. One has the following 
Proposition 6. Let $A_{0}$ be a constant with respect to $\xi$ and $\bar{\xi}$. Then the metric function $A e^{2 U}$ for the Ernst potential (2.12) is given by the expression:

$$
\left(A-A_{0}\right) e^{2 U}=-\rho\left(\frac{1}{Q} \frac{\Theta_{\mathbf{p q}}(0) \Theta_{\mathbf{p q}}\left(\int_{\xi}^{\infty^{-}}+\int_{\bar{\xi}}^{\infty^{-}}\right)}{\Theta_{\mathbf{p q}}\left(\int_{\xi}^{\infty^{-}}\right) \Theta_{\mathbf{p q}}\left(\int_{\bar{\xi}}^{\infty^{-}}\right)}-1\right)
$$

Proof. We have to show that equation (4.2) is satisfied with the function $A$ given by expression (4.4). It is convenient to introduce the auxiliary function $Z:=\left(A-A_{0}\right) e^{2 U}$; then equation (4.2) is obviously equivalent to the equation

$$
Z_{\bar{\xi}}=\frac{1}{\mathcal{E}+\overline{\mathcal{E}}}\left((Z+\rho) \overline{\mathcal{E}}_{\bar{\xi}}+(Z-\rho) \mathcal{E}_{\bar{\xi}}\right)
$$

The first step in the proof is to establish the relation

$$
\begin{aligned}
& 4\left(\ln \frac{\Theta_{\mathbf{p q}}\left(\int_{\xi}^{\infty^{-}}+\int_{\bar{\xi}}^{\infty^{-}}\right)}{\Theta\left(\int_{\bar{\xi}}^{\infty^{-}}\right)}\right)_{\bar{\xi}}=-3 c_{1}^{2}\left(\xi, \bar{\xi}, \infty^{-}\right) \\
& +c_{2}^{2}\left(\xi, \bar{\xi}, \infty^{-}\right)\left(4 Q \frac{\Theta_{\mathbf{p q}}\left(\int_{\xi}^{\infty^{-}}\right) \Theta_{\mathbf{p q}}\left(\int_{\bar{\xi}}^{\infty^{-}}\right)}{\Theta_{\mathbf{p q}}(0) \Theta_{\mathbf{p q}}\left(\int_{\xi}^{\infty^{-}}+\int_{\bar{\xi}}^{\infty^{-}}\right)}-1\right) \\
& +2 c_{2}\left(\xi, \bar{\xi}, \infty^{-}\right) \frac{\Theta_{\mathbf{p q}}\left(\int_{\xi}^{\infty^{-}}\right) \Theta_{\mathbf{p q}}\left(\int_{\xi}^{\infty^{-}}+\int_{\xi}^{\infty^{-}}\right)}{\Theta_{\mathbf{p q}}\left(\int_{\bar{\xi}}^{\infty^{-}}\right) \Theta_{\mathbf{p q}}\left(\int_{\xi}^{\infty^{-}}+\int_{\bar{\xi}}^{\infty^{-}}\right)} D_{\bar{\xi}} \ln \Theta_{\mathbf{p q}}(0)
\end{aligned}
$$

The proof of this statement follows step by the step the proof of proposition 3 . Using (2.16), we get for the l.h.s. of (4.6)

$$
\begin{aligned}
& D_{\bar{\xi}} D_{\bar{\xi}} \ln \frac{\Theta_{\mathbf{p q}}\left(\int_{\xi}^{\infty^{-}}+\int_{\bar{\xi}}^{\infty^{-}}\right)}{\Theta\left(\int_{\bar{\xi}}^{\infty^{-}}\right)} \\
& +D_{\bar{\xi}} \ln \frac{\Theta_{\mathbf{p q}}\left(\int_{\xi}^{\infty^{-}}+\int_{\bar{\xi}}^{\infty^{-}}\right)}{\Theta\left(\int_{\bar{\xi}}^{\infty^{-}}\right)} D_{\bar{\xi}} \ln \left(\Theta_{\mathbf{p q}}\left(\int_{\xi}^{\infty^{-}}+\int_{\bar{\xi}}^{\infty^{-}}\right) \Theta\left(\int_{\bar{\xi}}^{\infty^{-}}\right)\right) \\
& \quad+c_{1}\left(\infty^{-}, \bar{\xi}, \infty^{+}\right)\left(2 D_{\bar{\xi}} \ln \Theta_{\mathbf{p q}}\left(\int_{\xi}^{\infty^{-}}+\int_{\bar{\xi}}^{\infty^{-}}\right)-D_{\bar{\xi}} \ln \Theta\left(\int_{\bar{\xi}}^{\infty^{-}}\right)\right) .
\end{aligned}
$$

With the help of degenerated Fay's identity (2.6) with $a=\xi, b=\bar{\xi}, c=\infty^{-}$and $\mathbf{z}=\int_{\bar{\xi}}^{\infty^{-}}$, its $D_{\bar{\xi}}$ derivative and the formula (3.19), we can rewrite the expression 
(4.7) as follows:

$$
\begin{array}{r}
c_{2}\left(\xi, \bar{\xi}, \infty^{-}\right) \frac{\Theta_{\mathbf{p q}}\left(\int_{\xi}^{\infty^{-}}\right) \Theta_{\mathbf{p q}}\left(\int_{\xi}^{\infty^{-}}+\int_{\xi}^{\infty^{-}}\right)}{\Theta_{\mathbf{p q}}\left(\int_{\bar{\xi}}^{\infty^{-}}\right) \Theta_{\mathbf{p q}}\left(\int_{\xi}^{\infty^{-}}+\int_{\bar{\xi}}^{\infty^{-}}\right)} D_{\bar{\xi}} \ln \left(\Theta_{\mathbf{p q}}\left(\int_{\xi}^{\infty^{-}}\right) \Theta_{\mathbf{p q}}\left(2 \int_{\xi}^{\infty^{-}}\right)\right) \\
-3 c_{1}\left(\xi, \bar{\xi}, \infty^{-}\right)\left(c_{1}\left(\xi, \bar{\xi}, \infty^{-}\right)+c_{2}\left(\xi, \bar{\xi}, \infty^{-}\right) \frac{\Theta_{\mathbf{p q}}\left(\int_{\xi}^{\infty^{-}}\right) \Theta_{\mathbf{p q}}\left(\int_{\xi}^{\infty^{-}}+\int_{\xi}^{\infty^{-}}\right)}{\Theta_{\mathbf{p q}}\left(\int_{\bar{\xi}}^{\infty^{-}}\right) \Theta_{\mathbf{p q}}\left(\int_{\xi}^{\infty^{-}}+\int_{\bar{\xi}}^{\infty^{-}}\right)}\right) .
\end{array}
$$

The theta derivatives in the first line of (4.8) can be related to derivatives of the theta function with zero argument via degenerated Fay's identity (2.6) for $a=\xi$, $b=\bar{\xi}, c=\infty^{-}, \mathbf{z}=0$ and $a=\infty^{+}, b=\bar{\xi}, c=\infty^{-}, \mathbf{z}=0$ respectively (note that $\left.\int_{\xi}^{\infty^{-}}+\int_{\xi}^{\infty^{-}}=\int_{\infty^{+}}^{\infty^{-}}\right)$. The resulting expression can be simplified using (3.23) and Fay's identity (2.6) with $\mathbf{z}=\int_{\xi}^{\infty^{-}}, a=\xi, b=\bar{\xi}, c=\infty^{-}, d=\infty^{+}$,

$$
\begin{aligned}
\Theta_{\mathbf{p q}}\left(\int_{\xi}^{\infty^{-}}+\int_{\bar{\xi}}^{\infty^{-}}\right) \Theta_{\mathbf{p q}}(0)+\Theta_{\mathbf{p q}}\left(2 \int_{\xi}^{\infty^{-}}\right) \Theta_{\mathbf{p q}}\left(\int_{\xi}^{\bar{\xi}}\right) & \\
& =2 Q \Theta_{\mathbf{p q}}\left(\int_{\xi}^{\infty^{-}}\right) \Theta_{\mathbf{p q}}\left(\int_{\bar{\xi}}^{\infty^{-}}\right)
\end{aligned}
$$

to $(4.6)$.

Furthermore, with the help of relations (3.17) and (3.24), we transform this expression as follows:

$$
\begin{gathered}
\ln \left(\frac{\Theta_{\mathbf{p q}}(0) \Theta_{\mathbf{p q}}\left(\int_{\xi}^{\infty^{-}}+\int_{\bar{\xi}}^{\infty^{-}}\right)}{Q \Theta_{\mathbf{p q}}\left(\int_{\xi}^{\infty^{-}}\right) \Theta_{\mathbf{p q}}\left(\int_{\bar{\xi}}^{\infty^{-}}\right)}\right)_{\bar{\xi}} \\
=c_{2}^{2}\left(\xi, \bar{\xi}, \infty^{-}\right)\left(Q \frac{\Theta_{\mathbf{p q}}\left(\int_{\xi}^{\infty^{-}}\right) \Theta_{\mathbf{p q}}\left(\int_{\bar{\xi}}^{\infty^{-}}\right)}{\Theta_{\mathbf{p q}}(0) \Theta_{\mathbf{p q}}\left(\int_{\xi}^{\infty^{-}}+\int_{\bar{\xi}}^{\infty^{-}}\right)}-1\right) \\
+\frac{c_{2}\left(\xi, \bar{\xi}, \infty^{-}\right)}{2} \frac{\Theta_{\mathbf{p q}}\left(\int_{\xi}^{\infty^{-}}\right) \Theta_{\mathbf{p q}}\left(\int_{\xi}^{\infty^{-}}+\int_{\xi}^{\infty^{-}}\right)}{\Theta_{\mathbf{p q}}\left(\int_{\bar{\xi}}^{\infty^{-}}\right) \Theta_{\mathbf{p q}}\left(\int_{\xi}^{\infty^{-}}+\int_{\bar{\xi}}^{\infty^{-}}\right)} D_{\bar{\xi}} \ln \Theta_{\mathbf{p q}}(0) \\
-\frac{c_{2}\left(\xi, \bar{\xi}, \infty^{-}\right)}{2} \frac{\Theta_{\mathbf{p q}}\left(\int_{\xi}^{\bar{\xi}}\right) \Theta_{\mathbf{p q}}\left(\int_{\bar{\xi}}^{\infty^{-}}\right)}{\Theta_{\mathbf{p q}}(0) \Theta_{\mathbf{p q}}\left(\int_{\xi}^{\infty^{-}}\right)} D_{\bar{\xi}} \ln \Theta_{\mathbf{p q}}\left(\int_{\xi}^{\bar{\xi}}\right)
\end{gathered}
$$


Taking into account the relation (3.23), this implies the following relation:

$$
\begin{aligned}
Z_{\bar{\xi}}= & \frac{c_{2}\left(\xi, \bar{\xi}, \infty^{-}\right) \rho}{2 Q} \frac{\Theta_{\mathbf{p q}}(0) \Theta_{\mathbf{p q}}\left(\int_{\xi}^{\infty^{-}}+\int_{\xi}^{\infty^{-}}\right)}{\Theta_{\mathbf{p q}}^{2}\left(\int_{\bar{\xi}}^{\infty^{-}}\right)} D_{\bar{\xi}} \ln \Theta_{\mathbf{p q}}(0) \\
& -\frac{c_{2}\left(\xi, \bar{\xi}, \infty^{-}\right) \rho}{2 Q} \frac{\Theta_{\mathbf{p q}}\left(\int_{\xi}^{\bar{\xi}}\right) \Theta_{\mathbf{p q}}\left(\int_{\bar{\xi}}^{\infty^{-}}+\int_{\xi}^{\infty^{-}}\right)}{\Theta_{\mathbf{p q}}^{2}\left(\int_{\xi}^{\infty^{-}}\right)} D_{\bar{\xi}} \ln \Theta_{\mathbf{p q}}\left(\int_{\xi}^{\bar{\xi}}\right) .
\end{aligned}
$$

Whereas the expression for $Z-\rho$ follows directly from (4.4), we can write $Z+\rho$, using Fay's identity (4.9), in the convenient form

$$
Z+\rho=\frac{\rho}{Q} \frac{\Theta_{\mathbf{p q}}\left(2 \int_{\xi}^{\infty^{-}}\right) \Theta_{\mathbf{p q}}\left(\int_{\xi}^{\bar{\xi}}\right)}{\Theta_{\mathbf{p q}}\left(\int_{\xi}^{\infty^{-}}\right) \Theta_{\mathbf{p q}}\left(\int_{\bar{\xi}}^{\infty^{-}}\right)}
$$

Relation (4.10) turns out to be equivalent to (4.5) if we use equalities (3.23), (3.5), (3.10) and (3.11).

4.2. Metric function $e^{2 k}$. The metric function $e^{2 k}$ was calculated in [14] as the $\tau$-function of the Schlesinger system associated to the Ernst equation. Here we shall prove the resulting formula using Fay's identities.

Theorem 3. The metric function $e^{2 k}$ is given by

$$
e^{2 k}=K \frac{\Theta_{\mathbf{p q}}(0) \Theta_{\mathbf{p q}}\left(\int_{\xi}^{\bar{\xi}}\right)}{\Theta(0) \Theta\left(\int_{\xi}^{\bar{\xi}}\right)} .
$$

where $K$ is a constant, and where as before $\int_{\xi}^{\bar{\xi}} \equiv-\frac{1}{2}(1, \ldots, 1)$.

Proof. We have to show that (4.3) is satisfied with $k$ given by (4.12). Taking into account the relations $(3.10),(3.11)$, and (3.23), we obtain the following proposition we need to prove:

Proposition 7. The following identity holds:

$$
\begin{gathered}
\frac{1}{8}\left(D_{\xi} D_{\xi} \ln \frac{\Theta_{\mathbf{p q}}(0) \Theta_{\mathbf{p q}}\left(\int_{\xi}^{\bar{\xi}}\right)}{\Theta(0) \Theta\left(\int_{\xi}^{\bar{\xi}}\right)}+\left(D_{\xi} \ln \Theta_{\mathbf{p q}}(0)\right)^{2}+\left(D_{\xi} \ln \Theta_{\mathbf{p q}}\left(\int_{\xi}^{\bar{\xi}}\right)\right)^{2}\right) \\
=\frac{1}{4} D_{\xi} \ln \Theta_{\mathbf{p q}}(0) D_{\xi} \ln \Theta_{\mathbf{p q}}\left(\int_{\xi}^{\bar{\xi}}\right) .
\end{gathered}
$$

Proof. As the first step of the proof of identity (4.13) we observe that (4.13) can be rewritten in terms of the theta-function without characteristics as follows:

$$
D_{\xi} D_{\xi} \ln \frac{\Theta(\mathbf{V}) \Theta\left(\int_{\xi}^{\bar{\xi}}+\mathbf{V}\right)}{\Theta(0) \Theta\left(\int_{\xi}^{\bar{\xi}}\right)}+\left(D_{\xi} \ln \Theta(\mathbf{V})\right)^{2}+\left(D_{\xi} \ln \Theta\left(\int_{\xi}^{\bar{\xi}}+\mathbf{V}\right)\right)^{2}
$$




$$
=2 D_{\xi} \ln \Theta(\mathbf{V}) D_{\xi} \ln \Theta\left(\int_{\xi}^{\bar{\xi}}+\mathbf{V}\right),
$$

where $\mathbf{V} \equiv \mathbf{B p}+\mathbf{q}$ i.e. all exponential terms arising from relation (2.2) between the theta-function with characteristics and the theta-function with shifted argument drop out; therefore the statement (4.13) takes the form (4.14).

The idea of the proof of identity (4.14) is the following: we define a function $F$ as the difference of the left-hand and the right-hand side of (4.14). We show that the derivatives of the function $F$ with respect to any components $p_{\alpha}$ and any $q_{\alpha}$ of vectors $\mathbf{p}$ and $\mathbf{q}$ vanish. Then function $F$ must be a constant with respect to $\mathbf{p}$ and $\mathbf{q}$; thus it is sufficient to observe that this function vanishes at $\mathbf{p}=\mathbf{q}=0$.

Function $F$ depends only on the combination $\mathbf{V} \equiv \mathbf{B p}+\mathbf{q}$; therefore, all partial derivatives of $F$ with respect to each $p_{\alpha}$ are linear combinations of the partial derivatives with respect to $q_{\alpha}$; thus it is sufficient to prove that all partial derivatives of $F$ with respect to $q_{\alpha}$ vanish.

In turn, to show that all partial derivatives of $F$ with respect to $q_{\alpha}$ are equal to zero, it is sufficient to prove that $D_{b} F \equiv \sum_{\alpha=1}^{g} \frac{\partial F}{\partial q_{\alpha}} \omega_{\alpha}(b) / d \tau_{b}$ vanishes for an arbitrary point $b \in \mathcal{L}$, taking into account the following lemma:

Lemma 5. There exists a positive divisor $b_{1}+\cdots+b_{g}$ of degree $g$ on $\mathcal{L}$ such that the vectors $\omega\left(b_{1}\right) / d \tau_{b_{1}}, \ldots, \omega\left(b_{g}\right) / d \tau_{b_{g}}$ are linearly independent.

Proof. Suppose the opposite, i.e. that $\operatorname{det}\left\{\omega_{\alpha}\left(b_{\beta}\right)\right\}$ vanishes for any divisor $b_{1}+$ $\cdots+b_{g}$. Let us integrate this determinant along a basic cycle $a_{\beta}$ with respect to variable $b_{\beta}$ for each $\beta$. On one hand, the result should equal 0 according to our assumption. On the other hand, we get the determinant of the unit matrix, which equals 1 . This contradiction proves the lemma. ${ }^{2}$

Thus for suitably chosen $b$, the vector $\omega(b) / d \tau_{b}$ will take all values in $\mathbb{C}^{g}$. If one can show that $D_{b} F=0$ for arbitrary $b$, this implies that $F$ must be a constant.

Now let us calculate the $D_{b}$ derivative of $F(4.14)$ where $b \neq \xi$ is an otherwise arbitrary point on $\mathcal{L}$. With the help of Fay's identity $(2.9)$ we can write down this derivative as follows:

$$
\begin{aligned}
D_{b} F & =d_{2}(b, \xi) \frac{\Theta\left(\int_{\xi}^{b}+\mathbf{V}\right) \Theta\left(\int_{b}^{\xi}+\mathbf{V}\right)}{\Theta^{2}(\mathbf{V})} D_{\xi} \ln \frac{\Theta\left(\int_{\xi}^{b}+\mathbf{V}\right) \Theta\left(\int_{b}^{\xi}+\mathbf{V}\right)}{\Theta^{2}\left(\int_{\xi}^{\bar{\xi}}+\mathbf{V}\right)} \\
& +d_{2}(b, \xi) \frac{\Theta\left(\int_{\bar{\xi}}^{b}+\mathbf{V}\right) \Theta\left(\int_{b}^{\bar{\xi}}+\mathbf{V}\right)}{\Theta^{2}\left(\int_{\xi}^{\bar{\xi}}+\mathbf{V}\right)} D_{\xi} \ln \frac{\Theta\left(\int_{\bar{\xi}}^{b}+\mathbf{V}\right) \Theta\left(\int_{b}^{\bar{\xi}}+\mathbf{V}\right)}{\Theta^{2}(\mathbf{V})}
\end{aligned}
$$

\footnotetext{
2It was noticed by the referee that this lemma also has a geometrical interpretation: it means that the canonical model of the curve is not contained in any hyperplane.
} 
The degenerated Fay identity (2.6) implies

$$
D_{\xi} \ln \left\{\Theta\left(\int_{\xi}^{b}+\mathbf{V}\right) \Theta\left(\int_{b}^{\xi}+\mathbf{V}\right)\right\}
$$

$$
=2 D_{\xi} \ln \Theta\left(\int_{\xi}^{\bar{\xi}}+\mathbf{V}\right)+c_{2}(\bar{\xi}, \xi, b) \frac{\Theta(\mathbf{V})}{\Theta\left(\int_{\xi}^{\bar{\xi}}+\mathbf{V}\right)}\left(\frac{\Theta\left(\int_{\bar{\xi}}^{b}+\mathbf{V}\right)}{\Theta\left(\int_{\xi}^{b}+\mathbf{V}\right)}-\frac{\Theta\left(\int_{b}^{\bar{\xi}}+\mathbf{V}\right)}{\Theta\left(\int_{b}^{\xi}+\mathbf{V}\right)}\right) .
$$

Substituting (4.16) into (4.15), together with the corresponding relation for $D_{\xi}\left\{\Theta\left(\int_{\bar{\xi}}^{b}+\mathbf{V}\right) \Theta\left(\int_{b}^{\bar{\xi}}+\mathbf{V}\right)\right\}$, we find that the $D_{b}$ derivative of $F$ is identically zero for all $b \neq \xi$. Consequently, the difference $F$ between the r.h.s. and 1.h.s of (4.14) must be a constant with respect to the characteristics $[\mathbf{p}, \mathbf{q}]$. Considering the case $[\mathbf{p}, \mathbf{q}]=[0,0]$ we see that both sides of (4.3) are zero in this case. This completes the proof.

\section{Outlook}

In this paper we have presented a unified approach to theta functional solutions to the Ernst equation, i.e. to the stationary axisymmetric vacuum Einstein equations. Based on Fay's trisecant identity, its degenerations and Rauch's variational formulas for hyperelliptic Riemann surfaces, we proved the validity for formulas for the Ernst potential. The complete metric and the Ernst potential can be given explicitly in terms of theta functions. This explicit form free of derivatives of the metric made it possible in [12] to solve a boundary value problem for a relativistic dust disc in terms of a theta-functional Ernst potential. The description of the dust discs requires partial degeneration of the curve $\mathcal{L}$ and subsequent "condensation" of the double points, as was done in [14].

It is an open questions whether the methods outlined in this article can also be of direct use in the solution of boundary value problems as e.g. in the context of dust discs or black-hole disc systems. It would be interesting to extend this approach to the Einstein-Maxwell case where the theta-functional solutions are given on non-hyperelliptic Riemann surfaces (see [10]).

\section{Acknowledgements}

CK thanks for financial support by the Schloessmann foundation. Research of DK was supported by the grant of Fonds pour la Formation de Chercheurs et l'Aide a la Recherche de Quebec and the grant of Natural Sciences and Engineering Research Council of Canada. We thank the anonymous referee for several useful suggestions.

\section{References}

[1] Dubrovin, B. A., Matveev, V.B., Novikov, S.P., Non-linear equations of Korteveg-deVries type, finite-zone linear operators, and Abelian varieties, (Russian) Uspehi Mat. Nauk 31 (1976), no. 1 (187), 55-136; translation in Russian Math. Surveys 31 (1976), $59-146$. 
[2] Its, A. R., Matveev, V.B., Schrödinger operators with the finite-band spectrum and the $N$-soliton solutions of the Korteweg-de Vries equation, (Russian) Teoret. Mat. Fiz. 23 (1975), no. 1, 51-68.

[3] Krichever, I. M., The averaging method for two-dimensional "integrable" equations, (Russian) Funktsional. Anal. i Prilozhen. 22 (1988), no. 3, 37-52, 96; translation in Funct. Anal. Appl. 22 (1988), no. 3, 200-213 (1989)

[4] Shiota, T., Characterization of Jacobian varieties in terms of soliton equations, Invent. Math. 83 (1986), no. 2, 333-382.

[5] Mumford, D., Tata Lectures on Theta. I and II. Progress in Mathematics, 28 and 43, respectively. Birkhäuser Boston, Inc., Boston, MA, 1983 and 1984.

[6] Fay, John D., Theta-functions on Riemann surfaces, Lecture Notes in Mathematics, Vol. 352. Springer-Verlag, Berlin-New York, 1973.

[7] Belinskii, V.A., Zakharov, V.E., Integration of the Einstein equations by means of the inverse scattering problem technique and construction of exact soliton solutions, Soviet Phys. JETP 48 (1978), no. 6, 985-994; translated from Zh. Èksper. Teoret. Fiz. 75 (1978), no. 6, 1955-1971 (Russian)

[8] Maison, D., Are the stationary axially symmetric Einstein equations completely integrable? Phys. Rev. Lett. 41 (1978), no. 8, 521-522.

[9] Kramer, D., Stephani, H., Herlt, E., MacCallum, M., Exact solutions of Einstein's field equations, Edited by Ernst Schmutzer. Cambridge Monographs on Mathematical Physics. Cambridge University Press, Cambridge-New York, 1980.

[10] Korotkin, D., Finite-gap solutions of the stationary axially symmetric Einstein equation in vacuo, (Russian) Teoret. Mat. Fiz. 77 (1988), no. 1, 25-41; translation in Theoret. and Math. Phys. 77 (1988), no. 1, 1018-1031 (1989)

[11] Neugebauer, G., Meinel, R., General relativistic gravitational field of the rigidly rotating disk of dust: Solution in terms of ultraelliptic functions, Phys. Rev. Lett. 75 (1995), no. 17, 3046-3047.

[12] Klein, C. and Richter, O., Exact relativistic gravitational field of a stationary counterrotating dust disk, Phys. Rev. Lett. 83 (1999), no. 15, 2884-2887.

[13] Klein, C. and Richter, O., Physically realistic solutions to the Ernst Equation on hyperelliptic Riemann surfaces, Phys. Rev. D (3) 58 (1998), no. 12, 124018.

[14] Korotkin, D., Matveev, V., On theta-function solutions of the Schlesinger system and the Ernst equation, (Russian) Funktsional. Anal. i Prilozhen. 34 (2000), no. 4, 18-34, 96; translation in Funct. Anal. Appl. 34 (2000), no. 4, 252-264.

[15] Rauch, H.E., Weierstrass points, branch points and moduli of Riemann surfaces, Comm. Pure Appl. Math. 12 (1959) 543-560.

Max-Planck-Institut für Physik, Föhringer Ring 6, 80805 München, Germany. E-mail address: klein@mppmu.mpg.de

Laboratoire de l'Univers et de ses Theories (LUTH) CNRS, Observatoire de Paris, F-92195 Meudon Cedex, France.

E-mail address: Christian.Klein@obspm.fr

Department of Mathematics and Statistics, Concordia University, 7141 SherBrook West, Montreal H4B 1R6 Quebec, Canada.

E-mail address: korotkin@mathstat.concordia.ca

Department of Mathematics and Statistics, Concordia University, 7141 SherBrook West, Montreal H4B 1R6 Quebec, Canada.

E-mail address: vasilisa@mathstat.concordia.ca 